\title{
AIRCRAFT PITCH ATTITUDE ADAPTIVE CONTROL VIA SINGULAR PERTURBATION TECHNIQUE
}

\author{
V.D. Yurkevich \\ Novosibirsk State Technical University \\ 20 K. Marx Av., Novosibirsk 630092, Russia
}

\begin{abstract}
The problem of aircraft pitch attitude control is treated in the presence of uncertain aerodynamics. The proposed design methodology guarantees desired pitch attitude transient performance indices by inducing of two-time-scale motions in the closed-loop system where the controller dynamics is a singular perturbation with respect to the system dynamics. The singular perturbation method is used in order to get explicit expressions for evaluation of the controller parameters. Stability of fast-motion transients for a large range of aerodynamic characteristics variations is maintained due to a high-frequency-gain online identification and gain tuning that are incorporated in the control loop. Numerical example and simulation results are presented.
\end{abstract}

\section{INTRODUCTION}

Due to the complexity, variability, and uncertainties of aerodynamics, many different control techniques are used in order to design aircraft and missile control systems aimed to maintain transient performances in the presence of uncertain aerodynamics.

There are numerous approaches to flight control system design based, for instance, on the Nonlinear Inverse Dynamics (NID) method, feedback linearization, nonlinear $H_{\infty}$, linear matrix inequalities, $l_{1}$ optimal control, and linear quadratic regulator (LQR) [1-4]. In particular, control systems with sliding mode discussed in [5] and control systems with the highest derivative in feedback treated in $[6,7]$, are very powerful tools for aircraft and missile control system design under uncertainties. 
It is well known that throughout the huge set of flight control applications, the proportional-integral (PI) / proportional-integral-derivative (PID) controllers are extensively used. Problems of PI/PID control system analysis and design are discussed, for instance, in [8-10]. The majority of known design procedures of PI/PID controllers are applicable merely for stable linear systems. Usually, the well known tuning rules suggested in [11], or its various modifications are widely used for selection of controller parameters.

In order to fetch out the best PI or PID controllers in accordance with the assigned design objectives, a set of tuning rules, identification and adaptation schemes has been developed [8]. The main disadvantage for the most part of the existing procedures for PI or PID controller design is that the desired transient performances cannot be guaranteed in the presence of nonlinear plant parameter variations and unknown external disturbances. In order to overcome this disadvantage, the singular perturbation technique $[12,13]$ may be used for PI or PID controller design as was shown in $[14,15]$ where desired output transients are guaranteed by inducing of two-time-scale motions in the closed-loop system. Stability conditions imposed on the fast and slow modes and sufficiently large mode separation rate between fast and slow modes can ensure that the full-order closed-loop nonlinear system achieves the desired properties in such a way that the output transient performances are desired and insensitive to external disturbances and parameter variations of the system. The stability of fast-motion transients in the closed-loop system is provided by proper selection of controller parameters, while slow-motion transients correspond to the stable reference model of desired mapping from reference input into controlled output.

The critical point of the workability of the singular perturbation design methodology is that the controller parameters should be selected in accordance with the requirement on fast-motion transients stability and, moreover, the desired degree of time-scale separation between the fast and slow modes in the closed-loop system should be provided $[14,15]$.

However, the large variations of parameters of the system may give the disappearance of the two-time-scale structure of the trajectories in the closed-loop system. In order to maintain the two-time-scale structure of the trajectories in the closed-loop system and the desired damping of fast transients, an adaptive gain tuning scheme is proposed in this paper. As a result, the desired pitch attitude transient performance indices can be provided for significantly large range of aerodynamic characteristics variations.

The paper is organized as follows. First, a model of the aircraft longitudinal motion is defined. Second, the singular perturbation design methodology is highlighted for the purpose of aircraft pitch attitude control in the presence of uncertain aerodynamics. Third, the utmost importance of high-frequency-gain online identification and gain tuning in nonlinear control systems designed via singular perturbation technique is shown. Fourth, a gain tuning procedure and high-frequency-gain online identification procedure are introduced. Finally, sim- 
ulation results of the aircraft pitch attitude adaptive control system are presented as well.

\section{AIRCRAFT CONTROL PROBLEM}

\subsection{Aircraft Longitudinal Motion Model}

A set of variables introduced below is highlighted in Fig. 1 and in Table 1 as well as the block-diagram of the discussed aircraft pitch attitude control system is shown on Fig. 2.

The discussed approach to PID adaptive controller design for aircraft pitch attitude angle is treated based on the aircraft longitudinal motion model represented in [16]:

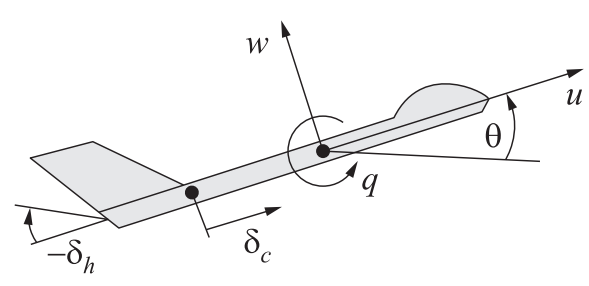

Figure 1 Aircraft longitudinal motion model

Table 1 Variables and parameters of the aircraft longitudinal motion model

\begin{tabular}{ll}
\hline \multicolumn{1}{c}{ Parameter } & \multicolumn{1}{c}{ Meaning } \\
\hline$a_{c}=20.0 \mathrm{~N} / \%$ & Coefficient \\
\hline$g=9.81 \mathrm{~m} / \mathrm{s}^{2}$ & Acceleration \\
\hline$J_{y}=5000 \mathrm{~kg} \cdot \mathrm{m}^{2}$ & Moment of inertia \\
\hline$L_{y}=0.5 \mathrm{~m}$ & $\begin{array}{l}\text { Equivalent arm force in the flow } \\
\text { coordinate system }\end{array}$ \\
\hline$m=2000 \mathrm{~kg}$ & Aircraft mass \\
\hline$q \in[-0.15,0.15] \mathrm{rad} / \mathrm{s}$ & Aircraft pitch rate \\
\hline$S_{x}=0.5 \mathrm{~m}^{2}, S_{y}=2.0 \mathrm{~m}^{2}$, & Equivalent areas in the flow coordinate \\
$S_{z}=10.0 \mathrm{~m}{ }^{2}$ & system \\
\hline$u \in[50,300] \mathrm{m} / \mathrm{s}$ & Longitudinal velocity \\
\hline$v_{a}=\left(v_{a x}^{2}+v_{a z}^{2}\right)^{1 / 2}$ & Air velocity value \\
\hline$v_{a x}=u-v_{w x} \cos \theta+v_{w z} \sin \theta$ & Longitudinal component of the air velocity \\
\hline$v_{a z}=w-v_{w x} \sin \theta-v_{w z} \cos \theta$ & Normal component of the air velocity \\
\hline$v_{w x} \in[-20,20] \mathrm{m} / \mathrm{s}$, & Components of wind velocity in the inertial \\
$v_{w z} \in[-10,10] \mathrm{m} / \mathrm{s}$ & system \\
\hline$w \in[-20,20] \mathrm{m} / \mathrm{s}$ & Normal velocity \\
\hline$\alpha=\tan -1\left(v_{a z} / v_{a x}\right)$ & Angle of attack \\
\hline$\delta_{c} \in[0 \%, 400 \%]$ & Thrust coefficient \\
\hline$\delta_{h} \in[-0.7,0.7] \mathrm{rad}$ & Angle of elevator deflection \\
\hline$\rho=1.2 \mathrm{~kg} / \mathrm{m}^{3}$ & Air density \\
\hline$\theta \in[-0.3,0.3] \mathrm{rad}$ & Aircraft pitch attitude angle \\
\hline
\end{tabular}




$$
\left.\begin{array}{l}
\dot{\theta}=q \\
\dot{u}=-w q-g \sin \theta+\frac{a_{c}}{m} \delta_{c}+\frac{1}{2 m} \rho v_{a}^{2}\left\{S_{x} c_{x}\left(\alpha, \delta_{h}\right) \cos \alpha-S_{z} c_{z}\left(\alpha, \delta_{h}\right) \sin \alpha\right\} \\
\dot{w}=u q+g \cos \theta+\frac{1}{2 m} \rho v_{a}^{2}\left\{S_{x} c_{x}\left(\alpha, \delta_{h}\right) \sin \alpha+S_{z} c_{z}\left(\alpha, \delta_{h}\right) \cos \alpha\right\} \\
\dot{q}=\frac{1}{2 J_{y}} \rho v_{a}^{2} L_{y} S_{y} m_{y}\left(\alpha, \delta_{h}\right) .
\end{array}\right\}
$$

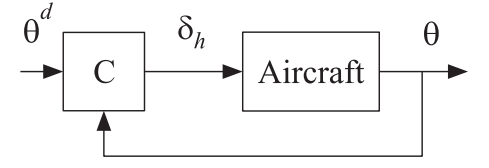

Figure 2 Block-diagram of control system

The functions $c_{x}\left(\alpha, \delta_{h}\right), \quad c_{z}\left(\alpha, \delta_{h}\right)$, and $m_{y}\left(\alpha, \delta_{h}\right)$ are approximated in the following form:

$$
\begin{aligned}
c_{x}\left(\alpha, \delta_{h}\right) & =c_{x}^{o}+c_{x}^{\alpha 2} \alpha^{2}+c_{x}^{h 2}\left(\delta_{h}\right)^{2} ; \\
c_{z}\left(\alpha, \delta_{h}\right) & =c_{z}^{o}+c_{z}^{\alpha} \alpha+c_{z}^{h} \delta_{h} ; \\
m_{y}\left(\alpha, \delta_{h}\right) & =m_{y}^{\alpha} \alpha+m_{y}^{h} \delta_{h}
\end{aligned}
$$

where $c_{x}^{o}=-0.2 ; c_{x}^{\alpha 2}=-0.002 ; c_{x}^{h 2}=-0.002 ; c_{z}^{h}=-10^{-4} ; c_{z}^{o}=-0.15$; $c_{z}^{\alpha}=-8.6 ; m_{y}^{\alpha}=0.057$; and $m_{y}^{h}=-0.01$.

\subsection{Problem of Aircraft Pitch Attitude Control}

The control problem is to provide the following condition:

$$
\lim _{t \rightarrow \infty} e_{\theta}(t)=0 \text {. }
$$

Here, $e_{\theta}(t)$ is the error of the reference input realization:

$$
e_{\theta}(t)=\theta^{d}(t)-\theta(t)
$$

where $\theta^{d}(t)$ is the reference input. Moreover, the controlled transients $e_{\theta}(t) \rightarrow 0$ should have the desired performance indices in the presence of external disturbances and varying parameters of the aircraft model (1).

\subsection{Input-Output Mapping of Aircraft Model}

From (1), it follows that the second time derivative of $\theta(t)$ depends algebraically on the control variable $\delta_{h}$, that is,

$$
\frac{d^{2} \theta}{d t^{2}}=f_{\theta}(\cdot)+b_{\theta}(\cdot) \delta_{h}
$$

where

$$
f_{\theta}(\cdot)=\frac{1}{2 J_{y}} \rho v_{a}^{2} L_{y} S_{y} m_{y}^{\alpha} \alpha ; \quad b_{\theta}(\cdot)=\frac{1}{2 J_{y}} \rho v_{a}^{2} L_{y} S_{y} m_{y}^{h}
$$


Hence, the second time derivative of $\theta(t)$ is the relative highest derivative of the output variable $\theta(t)$. Note, the condition $b_{\theta}(\cdot)<0$ holds for the above defined parameters.

Remark 1. Expression (3) describes the input-output mapping of the aircraft model without taking into account the internal dynamics of aircraft (in particular case, that are zero-dynamics). It is assumed through the text that the internal dynamics are stable or at least are bounded. The analysis of the internal behavior of system (1) was done in [17].

Remark 2. The parameter $b_{\theta}$ is called the high-frequency gain of the inputoutput mapping defined by (3) where $\theta$ is considered as the output variable, while $\delta_{h}$ is considered as the input variable.

Remark 3. The high-frequency gain $b_{\theta}$ may undergo extensive variations depending on operating point and aerodynamic characteristics, in particular, due to the variation of the air velocity $v_{a}$.

\section{CONTROLLER DESIGN VIA TIME-SCALE SEPARATION}

\subsection{Proportional-Integral-Derivative Controller}

Consider the controller given by

$$
\mu^{2} \delta_{h}^{(2)}+d_{1} \mu \delta_{h}^{(1)}=k\left[-\theta^{(2)}-a_{1} \theta^{(1)}+a_{0}\left[\theta^{d}-\theta\right]\right]
$$

where $\mu$ is the small positive parameter, $a_{0}>0$, and $a_{1}>0$. The parameters $a_{0}$ and $a_{1}$ are selected such that the polynomial

$$
s^{2}+a_{1} s+a_{0}
$$

has the desired root distribution inside the left part of the $s$-plane, where roots of the polynomial (5) are defined by the requirements imposed on the desired output transient performance indices of $\theta(t)$ in system (1).

Remark 4. The control law (4) can be expressed in terms of transfer functions that is the structure of PID controller with filter

$$
\delta_{h}(s)=\frac{k}{\mu\left(\mu s+d_{1}\right)}\left\{\frac{a_{0}}{s}\left[\theta^{d}(s)-\theta(s)\right]-\left(s+a_{1}\right) \theta(s)\right\}
$$

where the controller is proper and implemented without an ideal differentiation of $\theta(t)$ or $\theta^{d}(t)$. The implementation of controller (4) is highlighted in details in $[15]$. 
Denote $F\left(\theta^{(1)}, \theta, \theta^{d}\right)=-a_{1} \theta^{(1)}+a_{0}\left[\theta^{d}-\theta\right]$ and then the controller given by (4) can be rewritten, for short, in the following form:

$$
\mu^{2} \delta_{h}^{(2)}+d_{1} \mu \delta_{h}^{(1)}=k\left[F\left(\theta^{(1)}, \theta, \theta^{d}\right)-\theta^{(2)}\right] .
$$

\subsection{Two-Time-Scale Motions Analysis}

In accordance with Remark 1, let consider the closed-loop system equations that are given by (3) and (6). The replacement of $\theta^{(2)}$ in (6) by the right member of (3) yields the closed-loop system equations in the form:

$$
\left.\begin{array}{c}
\theta^{(2)}=f_{\theta}(\cdot)+b_{\theta}(\cdot) \delta_{h} \\
\mu^{2} \delta_{h}^{(2)}+d_{1} \mu \delta_{h}^{(1)}+k b_{\theta}(\cdot) \delta_{h}=k\left[F\left(\theta^{(1)}, \theta, \theta^{d}\right)-f_{\theta}(\cdot)\right] .
\end{array}\right\}
$$

Denote $\theta_{1}=\theta, \theta_{2}=\theta^{(1)}, \delta_{h 1}=\delta_{h}$, and $\delta_{h 2}=\mu \delta_{h}^{(1)}$. Hence, from (7), the singularly perturbed differential equations

$$
\left.\begin{array}{rl}
\dot{\theta}_{1} & =\theta_{2} ; \\
\dot{\theta}_{2} & =f_{\theta}(\cdot)+b_{\theta}(\cdot) \delta_{h 1} ; \\
\mu \dot{\delta_{h 1}} & =\delta_{h 2} ; \\
\mu \dot{\delta_{h 2}} & =-k b_{\theta}(\cdot) \delta_{h 1}-d_{1} \delta_{h 2}+k\left[F\left(\theta_{2}, \theta_{1}, \theta^{d}\right)-f_{\theta}(\cdot)\right]
\end{array}\right\}
$$

result as $\mu \rightarrow 0$.

If $\mu \rightarrow 0$, then fast and slow modes are artificially forced in system (8) where the time-scale separation between these modes depends on the parameter $\mu$. Hence, the properties of (8) can be analyzed on the basis of the two-time-scale technique and, as a result, slow and fast motion subsystems will be derived below and then analyzed separately.

Let introduce the new fast time scale $t_{0}=t / \mu$. Hence, from (8), the closedloop system equations in the new time scale $t_{0}$

$$
\left.\begin{array}{rl}
\frac{d}{d t_{0}} \theta_{1} & =\mu \theta_{2} ; \\
\frac{d}{d t_{0}} \theta_{2} & =\mu\left[f_{\theta}(\cdot)+b_{\theta}(\cdot) \delta_{h 1}\right] ; \\
\frac{d}{d t_{0}} \delta_{h 1} & =\delta_{h 2} ; \\
\frac{d}{d t_{0}} \delta_{h 2} & =-k b_{\theta}(\cdot) \delta_{h 1}-d_{1} \delta_{h 2}+k\left[F\left(\theta_{2}, \theta_{1}, \theta^{d}\right)-f_{\theta}(\cdot)\right]
\end{array}\right\}
$$

result. If $\mu \rightarrow 0$, then from (9), the fast-motion subsystem (FMS) equations 


$$
\begin{aligned}
\frac{d}{d t_{0}} \delta_{h 1} & =\delta_{h 2} \\
\frac{d}{d t_{0}} \delta_{h 2} & =-k b_{\theta}(\cdot) \delta_{h 1}-d_{1} \delta_{h 2}+k\left[F\left(\theta_{2}, \theta_{1}, \theta^{d}\right)-f_{\theta}(\cdot)\right]
\end{aligned}
$$

follow, where $d \theta_{1} / d t_{0}=0$ and $d \theta_{2} / d t_{0}=0$. Then, by returning to the primary time scale $t=\mu t_{0}$, the following FMS equations can be obtained:

$$
\left.\begin{array}{l}
\mu \dot{\delta_{h 1}}=\delta_{h 2} ; \\
\mu \dot{\delta_{h 2}}=-k b_{\theta}(\cdot) \delta_{h 1}-d_{1} \delta_{h 2}+k\left[F\left(\theta_{2}, \theta_{1}, \theta^{d}\right)-f_{\theta}(\cdot)\right]
\end{array}\right\}
$$

where $\theta_{1}$ and $\theta_{2}$ are treated as the frozen values during the transients in (10). This requirement can be easily satisfied for sufficiently small design parameter $\mu$. Finally, the FMS equations (10) may by rewritten as

$$
\mu^{2} \delta_{h}^{(2)}+d_{1} \mu \delta_{h}^{(1)}+k b_{\theta}(\cdot) \delta_{h}=k\left[F\left(\theta^{(1)}, \theta, \theta^{d}\right)-f_{\theta}(\cdot)\right]
$$

where $f_{\theta}(\cdot)$ and $b_{\theta}(\cdot)$ are treated as the constant values during the transients in (11).

Assume that the sign of $k$ is selected such that the condition $k b_{\theta}(\cdot)>0$ holds as well as the stability and desired fast damping of FMS transients are provided by selection of controller parameters $d_{1}, \mu$, and $k$. Then, the quasi-steady state for the FMS (11) yields $\delta_{h}(t)=\delta_{h}^{i d}(t)$ where

$$
\delta_{h}^{i d}=b_{\theta}^{-1}(\cdot)\left[F\left(\theta^{(1)}, \theta, \theta^{d}\right)-f_{\theta}(\cdot)\right]
$$

and $\delta_{h}^{\text {id }}$ is exactly the inverse dynamics solution. Substitution of $\delta_{h}=\delta_{h}^{\text {id }}$ into (3) yields the slow-motion subsystem (SMS) equation

$$
\theta^{(2)}+a_{1} \theta^{(1)}+a_{0} \theta=a_{0} \theta^{d}
$$

or, by another way, the SMS equation (12) can be directly derived from (8) by taking $\mu=0$.

In accordance with the main qualitative property of the singularly perturbed systems $[12,13,18,19]$, if an isolated equilibrium point of the FMS (11) exists and one is exponentially stable, then there exists $\mu^{*}>0$ such that for all $\mu \in\left(0, \mu^{*}\right)$, the trajectories of the singularly perturbed system (8) approximate to the trajectories of the SMS (12). So, if a sufficient time-scale separation between the fast and slow modes in system (8) and exponential convergence of FMS transients to equilibrium are provided, then, after the damping of fast transients, the desired output behavior prescribed by (12) is fulfilled despite that $f_{\theta}(\cdot)$ and $b_{\theta}(\cdot)$ are unknown complex functions. Thus, the output transient performance indices are insensitive to parameter variations of the nonlinear system and external disturbances; by that, the solution of the discussed control problem (2) is maintained. 


\section{ADAPTIVE PROPORTIONAL-INTEGRAL-DERIVATIVE CONTROLLER}

\subsection{Problem of High-Frequency-Gain Online Identification and Gain Tuning}

The critical point of the workability of the discussed design methodology via singular perturbation technique is that the controller parameters should be selected in accordance with the requirement on FMS stability and, moreover, the desired degree of time-scale separation between the fast and slow modes in the closed-loop system (8) should be provided.

From (11), the FMS characteristic polynomial

$$
A_{\mathrm{fms}}(s)=\mu^{2} s^{2}+d_{1} \mu s+\gamma
$$

follows, where $\gamma=k b_{\theta}(\cdot)$.

The main disadvantage is that the decrease of time-scale separation degree and loss of FMS transient performances as well as loss of FMS stability may occur in case of large variations of the high-frequency gain $b_{\theta}(\cdot)$.

In order to overcome the disadvantage caused by variations of $b_{\theta}$, two problems have to be solved. The first one is online identification of $b_{\theta}$. The second one is the adaptive gain tuning based on the knowledge of an estimate $\hat{b}_{\theta}$ for $b_{\theta}$. Then, by taking

$$
k=k_{1} \bar{k}_{0} ; \quad \bar{k}_{0}=\hat{b}_{\theta}^{-1}
$$

the condition $\gamma \approx k_{1}$ holds where $k_{1}>0$. As a result of gain tuning given by (13), the variations of the high-frequency gain $b_{\theta}$ do not alter the FMS transient performance indices.

\subsection{Adaptive Gain Tuning}

The block diagram of the proposed aircraft pitch attitude control system with adaptive gain tuning is shown in Fig. 3. Let take $\bar{k}_{0}=\bar{k} k_{0}$ and

$$
\delta_{h}=\bar{k} k_{0} \hat{\delta_{h}}
$$

where $\hat{\delta_{h}}$ is the new control variable; $\bar{k}=\operatorname{sgn}\left(b_{\theta}\right)=-1$; and $k_{0}>0$ is the tuning gain.

Consider the method of online identification based on a high-frequency probing signal [20]. Let

$$
\hat{\delta_{h}}(t)=\tilde{\delta_{h}}(t)+\delta_{h}^{0} \sin (\omega t)
$$




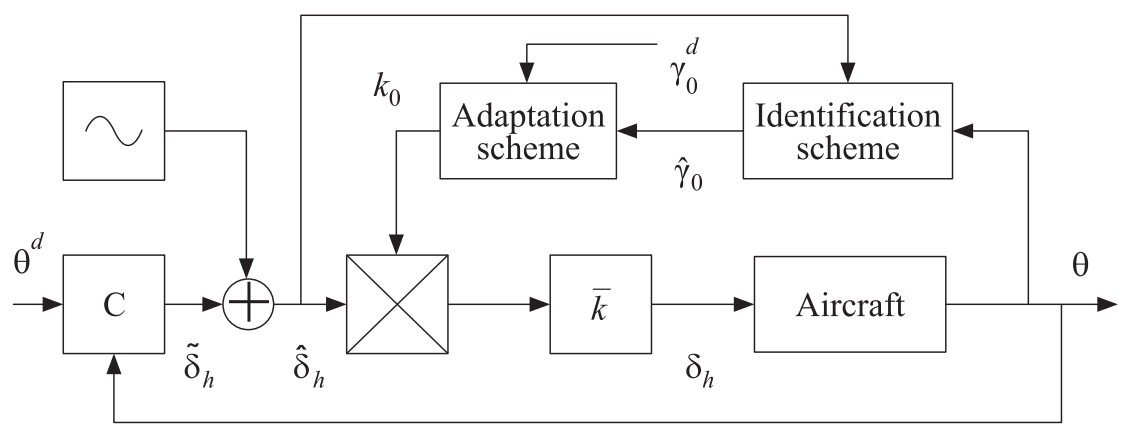

Figure 3 Block diagram of the aircraft pitch attitude control system with adaptive gain tuning

where $\delta_{h}^{0} \sin (\omega t)$ is the high-frequency probing signal with small value of amplitude $\delta_{h}^{0}$. Next, let rewrite the controller given by (6) as

$$
\mu^{2} \tilde{\delta}_{h}^{(2)}+d_{1} \mu \tilde{\delta}_{h}^{(1)}=k_{1}\left[F\left(\theta^{(1)}, \theta, \theta^{d}\right)-\theta^{(2)}\right] .
$$

Take, for example, the tuning rule in the following form:

$$
\frac{d k_{0}}{d t}=\alpha_{\gamma}\left[\gamma_{0}^{d}-\hat{\gamma}_{0}\right] ; \quad k_{0}(0)=k_{0}^{0}
$$

where $\gamma_{0}^{d}$ is the desired value of $\gamma_{0}$ and $\hat{\gamma}_{0}$ is the estimate of

$$
\gamma_{0}=\bar{k} k_{0} b_{\theta}(\cdot) \text {. }
$$

It is clear, in the case of steady-state when $d k_{0} / d t=0$, from (17) that the condition $k_{0}=\gamma_{0}^{d} /\left(\bar{k} \hat{b}_{\theta}(\cdot)\right)$ results, where $\hat{b}_{\theta}(\cdot)$ is the estimate of $b_{\theta}(\cdot)$.

\subsection{High-Frequency-Gain Online Identification}

The high-frequency small oscillations are forced in $\hat{\delta}_{h}(t)$ and $\theta(t)$ due to the highfrequency probing signal $\delta_{h}^{0} \sin (\omega t)$ that is incorporated in the control system as shown in (15). Let $A_{\hat{\delta}_{h}}$ be the amplitude of oscillations with frequency $\omega$ that are forced in the control $\hat{\delta}_{h}(t)$ and $A_{\theta}$ be the amplitude of oscillations with frequency $\omega$ that are forced in the output $\theta(t)$. From (3) and (14), the fundamental relation for the high-frequency-gain online identification follows, that is,

$$
\lim _{\omega \rightarrow \infty} \frac{A_{\theta}(\omega)}{A_{\hat{\delta}_{h}}(\omega)} \omega^{2}=\gamma_{0}
$$




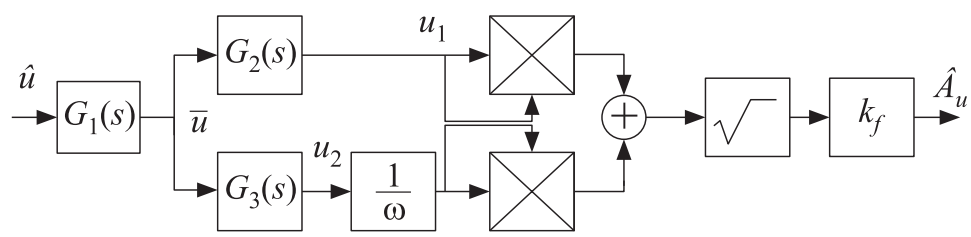

Figure 4 Block diagram of the amplitude detector

where $\gamma_{0}$ is defined by (18). Hence, the high-frequency-gain online identification scheme in Fig. 3 via relation (19) involves two amplitude detectors for $A_{\theta}(\omega)$ and $A_{\hat{\delta}_{h}}(\omega)$.

It is well known that if $\xi(t)=A_{\xi} \sin (\omega t)$, then

$$
A_{\xi}=\sqrt{\xi^{2}+\left(\frac{\dot{\xi}}{\omega}\right)^{2}} .
$$

The block-diagram of the amplitude detector shown in Fig. 4 is based on a practical implementation of $(20)$, where $\hat{u}$ is the complicated signal and $\hat{A}_{u}$ is the estimate of amplitude of oscillations with frequency $\omega$ in $\hat{u}$. The transfer functions $G_{1}(s), G_{2}(s)$, and $G_{3}(s)$ were selected as the following ones:

$$
\begin{gathered}
G_{1}(s)=\frac{\tau_{0}^{3} s^{3}}{\left(\tau_{0} s+1\right)^{3}} ; \quad G_{2}(s)=\frac{1}{\left(\tau_{f} s+1\right)^{3}} ; \quad G_{3}(s)=s G_{2}(s) ; \\
k_{f}=\sqrt{\left[1-3\left(\tau_{f} \omega\right)^{2}\right]^{2}+\left[\tau_{f} \omega\left(3-\left(\tau_{f} \omega\right)^{2}\right)\right]^{2}} ; \quad \hat{A}_{u}=k_{f} \sqrt{u_{1}^{2}+\left(u_{2} / \omega\right)^{2}} .
\end{gathered}
$$

The transfer function $G_{1}(s)$ is included in order to separate frequency between the processes of identification and input-output transient responses.

Two identical amplitude detectors shown in Fig. 4 were used where $\hat{u}=\hat{\delta}_{h}$ in the first one in order to get $\hat{A}_{\hat{\delta}_{h}}$ that is the estimate of $A_{\hat{\delta}_{h}}(\omega)$, whereas $\hat{u}(t)=\theta$ in the second one in order to get $\hat{A}_{\theta}$ that is the estimate of $A_{\theta}(\omega)$.

Finally, with the help of the low-pass filter given by

$$
\tau_{1} \frac{d \hat{\gamma}_{0}}{d t}+\hat{\gamma}_{0}=\frac{\hat{A}_{\theta}}{\hat{A}_{\hat{\delta}_{h}}+\epsilon} \omega^{2} ; \quad \hat{\gamma}_{0}(0)=\hat{\gamma}_{0}^{0},
$$

the estimate $\hat{\gamma}_{0}$ results, where $\epsilon$ is the small positive parameter in order to avoid the singularity condition in the right member of (21).

\section{SIMULATION RESULTS}

The simulation results of the closed-loop system are based on the aircraft longitudinal motion model (1) with (14). The high-frequency probing signal is 


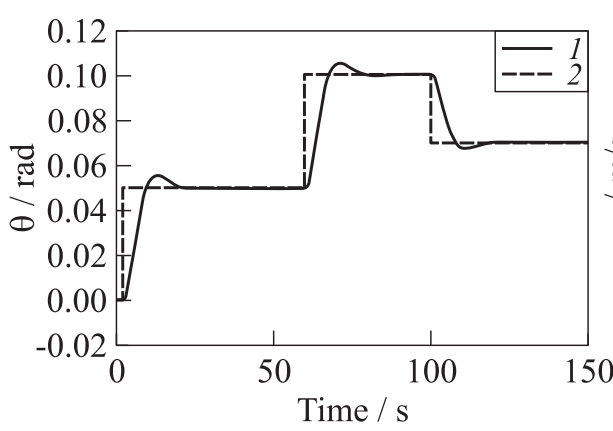

Figure 5 Plots of the aircraft pitch attitude $\theta(t)(1)$ and $\theta^{d}(t)(2)$

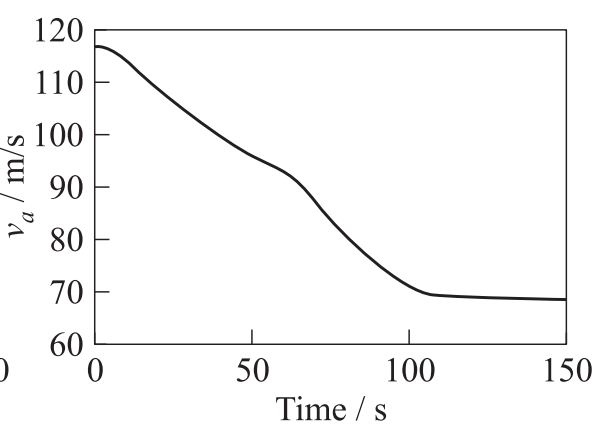

Figure 6 Plot of the air velocity $v_{a}(t)$

incorporated in the system as shown in (15). In accordance with (16), consider the feedback controller (C) in Fig. 3 in the following form:

$$
\mu^{2} \tilde{\delta}_{h}^{(2)}+d_{1} \mu \tilde{\delta}_{h}^{(1)}=k_{1}\left[-\theta^{(2)}-a_{1} \theta^{(1)}+a_{0}\left[\theta^{d}-\theta\right]\right] .
$$

The adaptive gain tuning for $k_{0}$ is provided in accordance with the rule (17) while the high-frequency-gain online identification is given by (21). The controller parameters are selected as $k_{1}=10, a_{0}=0.1145, a_{1}=0.4, d_{1}=1.9$, and $\mu=0.9347 \mathrm{~s}$. The parameters of the adaptive gain tuning scheme and the highfrequency-gain online identification scheme are selected as $\gamma_{0}^{d}=1, \hat{\gamma}_{0}(0)=1$, and $\epsilon=10^{-5}$. For all $t, \alpha_{\gamma}=100, k_{0}(0)=65, \omega=100 \mathrm{rad} / \mathrm{s}, A_{\omega}=0.0003$, $\tau_{f}=0.01 \mathrm{~s}, \tau_{0}=0.01 \mathrm{~s}, \tau_{1}=0.3 \mathrm{~s}$, and $\delta_{c}(t)=20 \%$. The simulation results of the closed-loop system are shown in Figs. 5-9. Figure 5 contains the plots of $\theta^{d}(t)$ and $\theta(t)$ showing the output step response in the closed-loop system where the air velocity $v_{a}(t)$ variations are displayed in Fig. 6. From Fig. 8, it can be observed that the condition $\hat{\gamma}_{0}(t) \rightarrow \gamma_{0}^{d}$ is kept due to the tuning of the gain $k_{0}(t)$ as seen in Fig. 9.

\section{CONCLUDING REMARKS}

The main advantage of the discussed singular perturbation technique for control system analysis and design is that the parameters of the PID adaptive controller for nonlinear aircraft model can be analytically derived in accordance with such indirect performance objectives as the desired root distribution of the reference model characteristic polynomial, while the desired root distribution is defined by such direct aircraft pitch attitude performance objectives as the settling time and overshoot. 


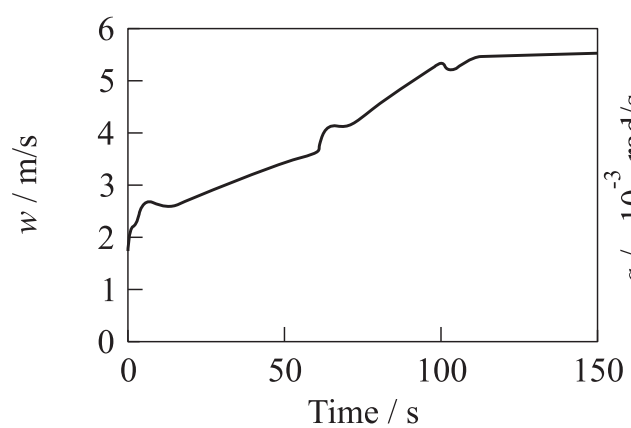

(a)

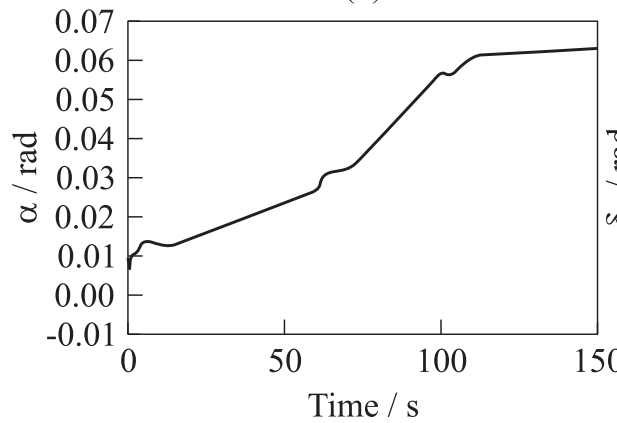

(c)

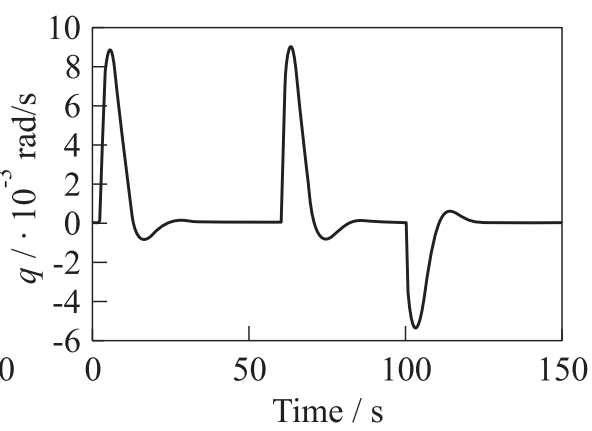

(b)

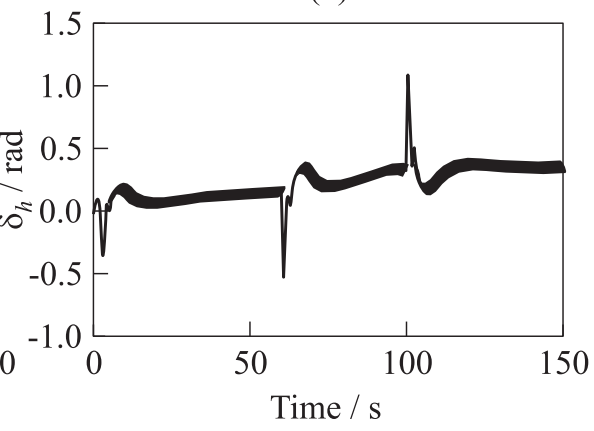

(d)

Figure 7 Plots of the normal velocity $w(t)(a)$; aircraft pitch rate $q(t)(b)$; angle of attack $\alpha(t)(c)$; and elevator deflection $\delta_{h}(t)(d)$

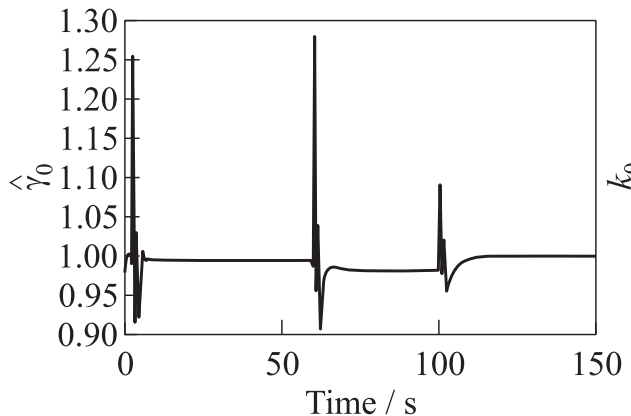

Figure 8 Plot of $\hat{\gamma}_{0}(t)$ where $\hat{\gamma}_{0}(t)$ is the estimate of $\gamma_{0}(t)$

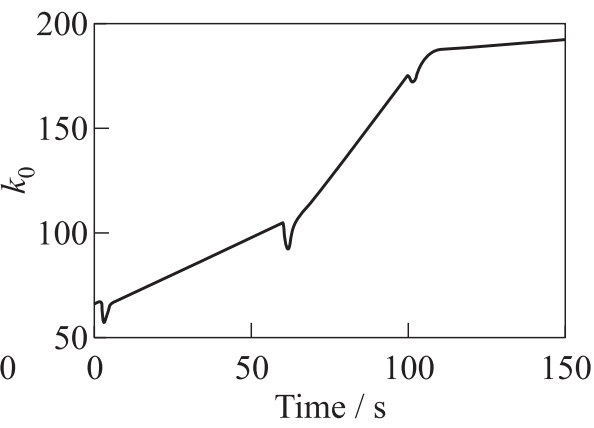

Figure 9 Plot of the tuning gain $k_{0}(t)$ 
The application of the singular perturbation technique in the presented design methodology allows to get desired aircraft pitch attitude transient performance indices for nonlinear aircraft model under uncomplete knowledge about external disturbances and aerodynamic characteristics. It has been shown that the highfrequency-gain online identification and gain tuning in the discussed adaptive control system allow to maintain the two-time scaling in the closed-loop system and desired transient performance indices of fast transients in case of significantly large range of aerodynamic characteristics variations. Simulation results show the effectiveness of the proposed control laws.

\section{REFERENCES}

1. Petrov, B. N., and P. D. Krutko. 1981. Inverse problems of controlled-flight dynamics: Lateral motion. Sov. Phys.-Dokl. 26(2):147-49.

2. Lane, S., and R. Stengel. 1988. Flight control design using non-linear inverse dynamics. Automatica 24:471-83.

3. Vukobratović, M., and R. Stojić. 1988. Modern aircraft flight control. Lecture notes in control and information sciences ser. Springer-Verlag. 109.

4. Wise, K. A. 1995. Applied controls research topics in the aerospace industry. 34th Conference on Decision and Control Proceedings. New Orleans, LA. 751-56.

5. Schumacher, D. A. 1994. Rapid response missile pitch to trim flight control using sliding modes. 33rd IEEE Conference on Decision and Control Proceedings. 4:385459 .

6. Błachuta, M. J., V.D. Yurkevich, and K. Wojciechowski. 1997. Design of analog and digital aircraft flight controllers based on dynamic contraction method. AIAA Guidance, Navigation, and Control Conference Proceedings. New Orleans, LA. 3:1719-29.

7. Czyba, R., and M. J. Błachuta. 2003. Robust longitudinal flight control design: Dynamic contraction method. American Control Conference Proceedings. Denver, Colorado. 1020-25.

8. Åström, K. J., T. Hagglund, C. C. Hang, and W. K. Ho. 1993. Automatic tuning and adaptation for PID controllers - a survey. Contr. Eng. Pract. 1(4):699-714.

9. Åström, K. J., and T. Hagglund. 1995. PID controllers: Theory, design, and tuning. Research Triangle Park, NC: Instrum. Soc. Amer.

10. O'Dwyer, A. 2003. Handbook of PI and PID tuning rules. London, UK: Imperial College Press.

11. Ziegel, G., and N.B. Nichols. 1942. Optimum settings for automatic controllers. Trans. ASME 64(8):759-68.

12. Kokotović, P. V., H. K. Khalil, and J. O'Reilly. 1999. Singular perturbation methods in control: Analysis and design. Philadelphia, PA: SIAM.

13. Naidu, D.S. 2002. Singular perturbations and time scales in control theory and applications: An overview. Dyn. Continuous Discrete Impulsive Syst. (DCDIS), Ser. B: Applications Algorithms 9(2):233-78. 
14. Yurkevich, V.D. 2004. Design of nonlinear control systems with the highest derivative in feedback. World Scientific.

15. Yurkevich, V.D. 2011. PI/PID control for nonlinear systems via singular perturbation technique. In: Advances in PID control. InTech. 113-42.

16. Wojciechowski, K., A. Ordys, and J. Polańska. 1989. Model Przestrzennego Ruchu Samolotu da Celow Symulacji i Sterowania. Zeszyty Naukowe Politechniki Slaskiej, 1989. [In Polish.]

17. Yurkevich, V. D., M. J. Błachuta, and K. Wojciechowski. 1991. Stabilization system for the aircraft longitudinal motion. Archiwum Automatyki i Robotyki. Warsaw. 36(3-4):517-35. [In Polish.]

18. Klimushchev, A.I., and N.N. Krasovskii. 1962. Uniform asymtotic stability of systems of differential equations with a small parameter in the derivative terms. J. Appl. Math. Mech. 25:1011-25.

19. Hoppensteadt, F.C. 1966. Singular perturbations on the infinite time interval. Trans. Amer. Math. Soc. 123:521-35.

20. Eykchoff, P. 1974. System identification. Wiley. 